\title{
Acute esophageal necrosis: possible association with terlipressin
}
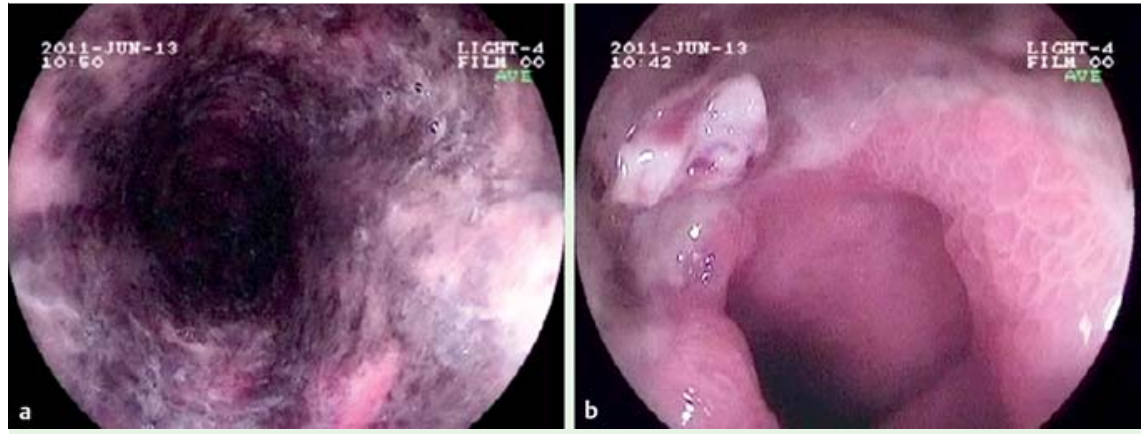

Fig. 1 Endoscopic views in a 75-year-old man with hematemesis and melena showing: a black mucosa consistent with acute esophageal necrosis; $\mathbf{b}$ an exposed vessel in an ulcer at the cardia.
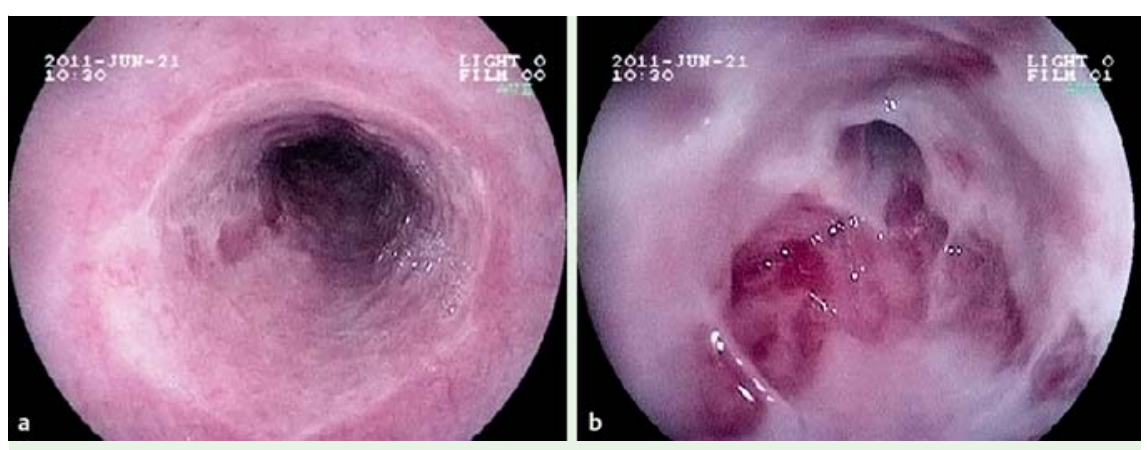

Fig. 2 Views during a second endoscopy performed after 8 days showing: a a clear margin between the intact proximal esophagus and the damaged middle third of the esophagus; $\mathbf{b}$ a stricture of the cardia.

A 75-year-old man was admitted to our department with abdominal pain, hematemesis, and melena. His significant medical history included erosive gastritis, alcohol-related chronic liver disease, and chronic pancreatitis. He was not receiving any medication. His blood pressure was low $(80 / 50 \mathrm{mmHg})$; results of laboratory testing showed macrocytic anemia and liver dysfunction (hemoglobin $11.8 \mathrm{~g} / \mathrm{dL}$, mean cell volume [MCV] 106.4fL, international normalized ratio [INR] 1.53). After a second episode of hematemesis, his hemoglobin dropped to $8.9 \mathrm{~g} / \mathrm{dL}$ and he was treated by infusion of a colloidal solution, two units of packed red blood cells, a proton pump inhibitor, and terlipressin ( $2 \mathrm{mg}$ every 4 hours).

Endoscopy showed a black mucosa $(\bullet$ Fig. 1 a) that started from the upper esophagus and ended abruptly at the cardia. At that level, we identified an ulcer ex- tending circumferentially in which there was a large exposed vessel ( $\bullet$ Fig. $\mathbf{1} \mathbf{b}$ ), which was treated by application of a Hemoclip. The stomach and duodenum were intact. Brushings were negative for cytomegalovirus. Broad-spectrum antibiotics, antifibrinolytic drugs, and parenteral nutrition were commenced; terlipressin was stopped.

Endoscopy at day 8 showed a clear margin between the intact proximal esophagus and its lower portion ( $\boldsymbol{O}$ Fig. $2 \mathrm{a}$ ). The luminal circumference decreased craniocaudally, ending in a stricture at the cardia ( $\bullet$ Fig.2b). At day 16, the distal esophagus appeared stenotic but was passable and enteral nutrition was resumed.

The patient was discharged 25 days after admission. A month later, endoscopy revealed almost complete restoration of the mucosa. Notably, at the cardia we observed a Schatzki ring ( $\bullet$ Fig. 3 ). A further

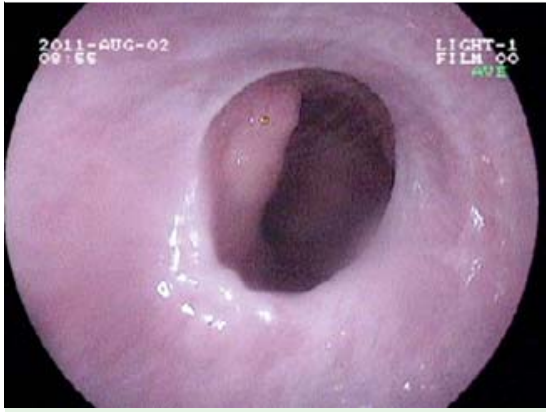

Fig. 3 View of the lower esophagus during an endoscopy performed a month after discharge showing a Schatzki ring.

endoscopy 8 months later showed no abnormal esophageal findings.

Acute esophageal necrosis is characterized by a circumferential mucosal blackening involving the distal esophagus and occasionally extending upstream that stops abruptly at the gastroesophageal junction [1]. Ulceration of the cardia, as in this case, is uncommon; however, similar cases have been reported [2].

Ischemia, impaired mucosal defenses, and chemical insult seem to contribute to its pathogenesis [3]. The distal esophagus has been shown to be less vascularized in angiographic studies [2,3], arguably making it susceptible to local hypoperfusion caused by low splanchnic blood flow. In the case described, such a state could have resulted from hemorrhage and hypotension.

Furthermore, because of the signs of liver dysfunction and the history of alcohol abuse, which suggested variceal bleeding, the patient received terlipressin, a splanchnic vasoconstrictor that may have reduced microcirculatory perfusion, further contributing to the local ischemia [4]. Although cutaneous necrosis following terlipressin treatment has been reported [5], this is the first reported case of a possible association with acute esophageal necrosis.

Endoscopy_UCTN_Code_CCL_1AB_2AC_3AH

Competing interests: None 
Konstantinos Efthymakis, Chiara Massacesi, Angelo Milano, Francesco Laterza, Emanuele Tafuri, Francesco Cipollone, Matteo Neri

Department of Medicine and Ageing Sciences, Section of Internal Medicine and Center for Excellence on Ageing (Ce.S.I.), “G. D’Annunzio” University and Foundation, Chieti, Italy

\section{References}

1 Moretó $M$, Ojembarrena E, Zaballa $M$ et al. Idiopathic acute esophageal necrosis: not necessarily a terminal event. Endoscopy 1993; 25: 534-538

2 Burtally A, Gregoire P. Acute esophageal necrosis and low-flow state. Can J Gastroenterol 2007; 21: 245-247

3 Gurvits GE. Black esophagus: acute esophageal necrosis syndrome. World J Gastroenterol 2010; 16: 3219-3225

4 Asfar P, Bracht $H$, Radermacher P. Impact of vasopressin analogues on the gut mucosal microcirculation. Best Pract Res Clin Anaesthesiol 2008; 22: 351-358

$5 \mathrm{Lu} \mathrm{YY}$, Wei KC, Wu CS. Terlipressin-induced extensive skin necrosis: a case report and published work review. J Dermatol 2012; 39: $866-868$

\section{Bibliography}

Dol http://dx.doi.org/

10.1055/s-0034-1365789

Endoscopy 2014; 46: E279-E280

(c) Georg Thieme Verlag KG

Stuttgart · New York

ISSN 0013-726X

\section{Corresponding author}

\section{Matteo Neri, MD}

U.O. di Gastroenterologia ed Endoscopia Digestiva Università G. D’Annunzio

Ospedale SS Annunziata

Chieti

Italy

Fax: +39-0871-357446

mneri@unich.it 